\title{
Physicians advice, parental practice and adherence to doctor's advice: an original survey on infant feeding
}

\author{
Jean-Pierre Chouraqui ${ }^{1 *}$ D, Bérénice Delmas ${ }^{2}$, Marine Le Bris ${ }^{2}$, Marc Bellaiche $^{3}$, Camille Jung $^{4}$ and Thierry Hanh ${ }^{2}$
}

\begin{abstract}
Background: Despite several years of guidance on infant feeding, there has been only a slight change in consumer compliance. Therefore, this study aimed to analyse parents feeding practices, explore physicians' advice about infant feeding and subsequent parent's adherence to advice.

Methods: A multicentric cross-sectional qualitative and descriptive self-report online study was conducted in volunteers to participate in the study.

Results: Fifty-four physicians (paediatricians and general practitioners) and 600 parents of infants were recruited. Of the infants, 20.2\% presented at inclusion with at least one type of functional gastrointestinal disorder. The breastfeeding prevalence was quite low (37.3\%). The main initial deviance from guidelines said they observed in infant feeding was the early use of cow's milk. More than two-thirds of infants older than 8 months were drinking cow's milk. The introduction of solid foods was globally in line with recommendations. Most physicians gave advice about the different aspects of infant feeding but were seeking more information, as did the parents. A discrepancy between the physicians' statements and the parents' perceptions was observed. However the majority (95.4\%) of parents reported that they followed totally or partially the advice received, especially by abandoning subsequently the use of cow's milk in favour of a formula. The main reason for not adhering to the advice was that they did not consider it suitable for their infant and they preferred to rely on their feelings or recommendations from familiars.

Conclusions: This survey provides good insights into parents' infant feeding practices together with the advice given by their doctor. The gap between practices and current guidelines is notable only for breast-feeding and use of formula. Despite several guidelines professionals and parents seek nutrition information. It highlights the need to deliver consistent, relevant, and less confusing messages about infant feeding.
\end{abstract}

Keywords: Adherence to physician's advice, Breastfeeding, Complementary foods, Evaluation of practices, Functional gastrointestinal disorders, Infant feeding practices, Infant formula

\section{Introduction}

Infant feeding is an important determinant of both growth and development and poses medium and longterm risks for developing non-communicable chronic diseases [1]. Different guidelines for feeding practices and nutrient requirements have been released over the years [2-8]. However, the general observation is that parents' dietary practices are incongruent with the guidelines [9-16]. A recent survey of 1184 families in

\footnotetext{
* Correspondence: chouraquijp@wanadoo.fr

${ }^{1}$ Paediatric Nutrition and Gastroenterology, Division of Pediatrics, Woman,

Mother and Child Department, Centre Hospitalier Universitaire Vaudois

(CHUV), 21 rue du Bugnon, 1011 Lausanne, Switzerland

Full list of author information is available at the end of the article
}

France highlighted the low prevalence and duration of breastfeeding and the early abandonment of infant formulas for the use of cow's milk, while the introduction of solid foods was done within the recommended time range [16]. While many factors may account for this gap [13], practitioners have a responsibility to provide evidence that is based on the best practice advice. Therefore, the aims of the present survey were: first to analyse the parents' prior practices in their infant's feeding, and to describe the subsequent physicians' advice on this feeding, and second to assess the parents' adherence to physician advice.

(c) The Author(s). 2019 Open Access This article is distributed under the terms of the Creative Commons Attribution 4.0 International License (http://creativecommons.org/licenses/by/4.0/), which permits unrestricted use, distribution, and reproduction in any medium, provided you give appropriate credit to the original author(s) and the source, provide a link to the Creative Commons license, and indicate if changes were made. The Creative Commons Public Domain Dedication waiver (http://creativecommons.org/publicdomain/zero/1.0/) applies to the data made available in this article, unless otherwise stated. 


\section{Materials and methods}

A multicentric cross-sectional qualitative and descriptive online study was conducted in mainland France from 5 February to 31 December 2016, according to the ethical standards.

\section{Participants}

All paediatricians and general practitioners (GPs) who were known to be regularly engaged in infant primary ambulatory care were invited by mail or email to participate in the study. Once agreed, they received a username and personal password to access a dedicated secure website (Optinutri.fr). Each practitioner had to invite parents of a first healthy infant aged 1 to 12 months to participate, provided they were fluent in French and able to use the Internet. The infants were divided into five age groups corresponding to the usual ages that the diet is modified: $1-2,3-4,5-6,8-9$, and 11-12 months (infants aged 7 or 10 months were excluded). After receiving verbal and written explanations of the purpose and content of the research parents gave first verbal consent and provided their e-mail address and telephone number, which were registered and encrypted on the website using a stream cipher (RC4, RSA Security LLC, MA, USA), and email and text messages were immediately sent to the parents: one with the link to the parent questionnaire and another with their personal access code. Parents confirmed their consent online when they first connected.

\section{Study design}

After baseline data were collected online, the physician interviewed the parents and examined the infant to complete the questionnaire. A remuneration equivalent to the price of an infant consultation was awarded for this work. Two weeks after this visit, the parents were contacted by email and text message and asked to access the website and complete their questionnaire, which was inaccessible to the physician.

\section{Questionnaires}

Online questionnaires were developed by the investigators using C\# (ASP.net) framework (version 2.0; Microsoft Corporation, WA, USA) together with Visual Studio software 2008 (Microsoft Corporation, Redmond, WA, USA). Data storage was externally managed using SQL server software (version 2008 R2; Microsoft Corporation, Redmond, WA, USA).

Both questionnaires were structured and consisted of multiple choice, yes/no, and open-ended questions (103 for physicians and 44 for parents) grouped into predefined sets. Physician questionnaires included (Additional file 1): birth and current infant characteristics; parents' allergy history, as defined by Kjellman [17]; functional gastrointestinal disorders (FGID) according to the Rome III criteria [18]; infant feeding practices since birth and currently, based on a $24 \mathrm{~h}$ recall; dietary advice given in relation to any breastfeeding, use of infant formula, the introduction of solid foods, or a possible FGID; and the time spent providing this advice. Parent questionnaires (Additional file 2) asked about infant feeding at follow-up, adherence to the doctor's advice, observance in their daily practice, and their feelings about the time devoted to this advice. After questionnaire completion, all parent identifiers were deleted from the data. At the end of the survey, the database was frozen after backup and access to the survey website was deactivated.

\section{Data analysis}

Results were given as the number, percentage, and median with the interquartile range (Q1-Q3). The Stat Process of SAS 9.2 (SAS Institute, Cary, NC, USA) was used for statistical analyses. Pearson's chi-squared or Fisher's exact tests with the Monte Carlo algorithm were used for the qualitative categorical and binary data, the Kruskal-Wallis test was used as a non-parametric test for the quantitative data. To compare doctor and parent's perception on the same item McNemar's test was used for the paired nominal data, and Cohen's kappa coefficient $(\mathrm{\kappa})$ measured inter-rater agreement for the qualitative (categorical) items. A significance level of 0.05 was used, with a $95 \%$ confidence interval.

\section{Results}

Of the contacted physicians, 159 (1.2\%) answered the invitation; 54 (34\%) physicians, including 31 paediatricians and 23 GPs, agreed to the protocol and conducted the study. The paediatricians included 461 infants (76.8\%) and the GPs included 139 infants (23.2\%). Three infants were excluded because they did not meet the inclusion criteria. The mother was the usual respondent (93.4\%).

\section{Data at inclusion \\ Infants' characteristics}

Infants' characteristics are shown in Table 1. A history of allergic disease was noted in $32.8 \%$ of the infants' parents (mother: $14.9 \%$; father: $12.4 \%$; both: $5.5 \%$ ).

\section{Functional gastrointestinal disorders}

Figure 1 presents the prevalence of FGID, which was higher in the GPs' practices (29.5\% vs $17.4 \%, p=0.003$ ). Simultaneous regurgitation and crying were noted in $7.8 \%$ of the infants, and constipation together with another FGID were present in $4.9 \%$ of the infants. Infants with regurgitation had 2.00 (1.00-4.00) regurgitation episodes per day (maximum 8.00). Excessive crying mainly occurred in the evening (57.9\%) and rarely during the night (15.8\%). Constipated infants had 4.00 (4.00-7.00) movements per week (minimum 1.00). The stools were 
Table 1 Infants' characteristics at inclusion, including gestational age, birth weight and length, and current age, weight, and length, given as the median with the interquartile range (Quartile 1 to Quartile 3)

\begin{tabular}{|c|c|c|c|c|c|}
\hline \multicolumn{6}{|c|}{ Age group (months) } \\
\hline $1-2$ & $3-4$ & $5-6$ & $8-9$ & $11-12$ & $\begin{array}{l}\text { Total } \\
N=600\end{array}$ \\
\hline
\end{tabular}

\begin{tabular}{|c|c|c|c|c|c|c|}
\hline \multicolumn{7}{|l|}{ Birth data } \\
\hline Number & 150 & 132 & 146 & 90 & 79 & 597 \\
\hline $\begin{array}{l}\text { Number (\%) of Preterm } \\
\text { infants }\end{array}$ & $7(4.7 \%)$ & 17 (12.9\%) & $13(8.9 \%)$ & $6(6.7 \%)$ & 7 (8.9\%) & $50(8.4 \%)$ \\
\hline Birth weight (kg) & 3.31 [3.05-3.57] & 3.28 [2.91-3.65] & 3.27 [3.00-3.50] & 3.27 [2.95-3.60] & 3.30r [3.00-3.55] & 3.29 [2.99-3.57] \\
\hline Birth length $(\mathrm{cm})$ & $49.70[48.00-51.00]$ & 50.00 [48.00-51.00] & $50.00[48.00-51.00]$ & $49.00[48.00-51.00]$ & 49.50 [48.00-51.00] & $50.00[48.00-51.00]$ \\
\hline \multicolumn{7}{|l|}{ Inclusion data } \\
\hline Number & 151 & 132 & 147 & 90 & 80 & 600 \\
\hline Sex ratio M/F & 1.40 & 1.28 & 1.10 & 0.91 & 1.22 & 1.19 \\
\hline Age (weeks) & 9.00 [4.00-9.00] & 17.00 [14.00-18.00] & $24.00[23.00-26.00]$ & 39.00 [37.00-40.00] & 50.00 [48.00-52.00] & $22.00[12.50-37.00]$ \\
\hline Weight (kg) & $4.90[4.15-5.40]$ & 6.51 [6.11-7.19] & 7.60 [7.00-8.30] & 8.76 [8.20-9.22] & $9.70[8.72-10.20]$ & 7.20 [5.78-8.50] \\
\hline Length $(\mathrm{cm})$ & $56.00[54.00-58.00]$ & $63.00[60.25-64.50]$ & $66.00[65.00-68.00]$ & 71.25 [69.00-73.00] & 75.00 [73.00-77.00] & $65.00[59.75-70.00]$ \\
\hline
\end{tabular}

described as hard in $60.7 \%$ of the infants and defecation was painful in $45.5 \%$.

\section{Feeding practices}

The prevalence of breastfeeding at the time of inclusion is depicted in Fig. 2a. No difference was observed between practitioners except in the 1-2 months age group (37.3\% of paediatricians vs $20.4 \%$ of GPs, $p=0.04$ ). Information was obtained about breastfeeding at birth for $63.7 \%$ of the current non-breastfed infants and there was a positive result in 21 infants. The subsequent estimated overall prevalence of breastfeeding at birth was $37.3 \%$. Problems with breastfeeding were reported by $16.7 \%$ of the breastfeeding mothers, which included ways to enhance lactation, engorgement or cracked nipples, having infants with a 'gourmet' or 'rester' feeding style, and/or planning their return to work. Overall, 286 (47.8\%) infants consumed a formula either exclusively

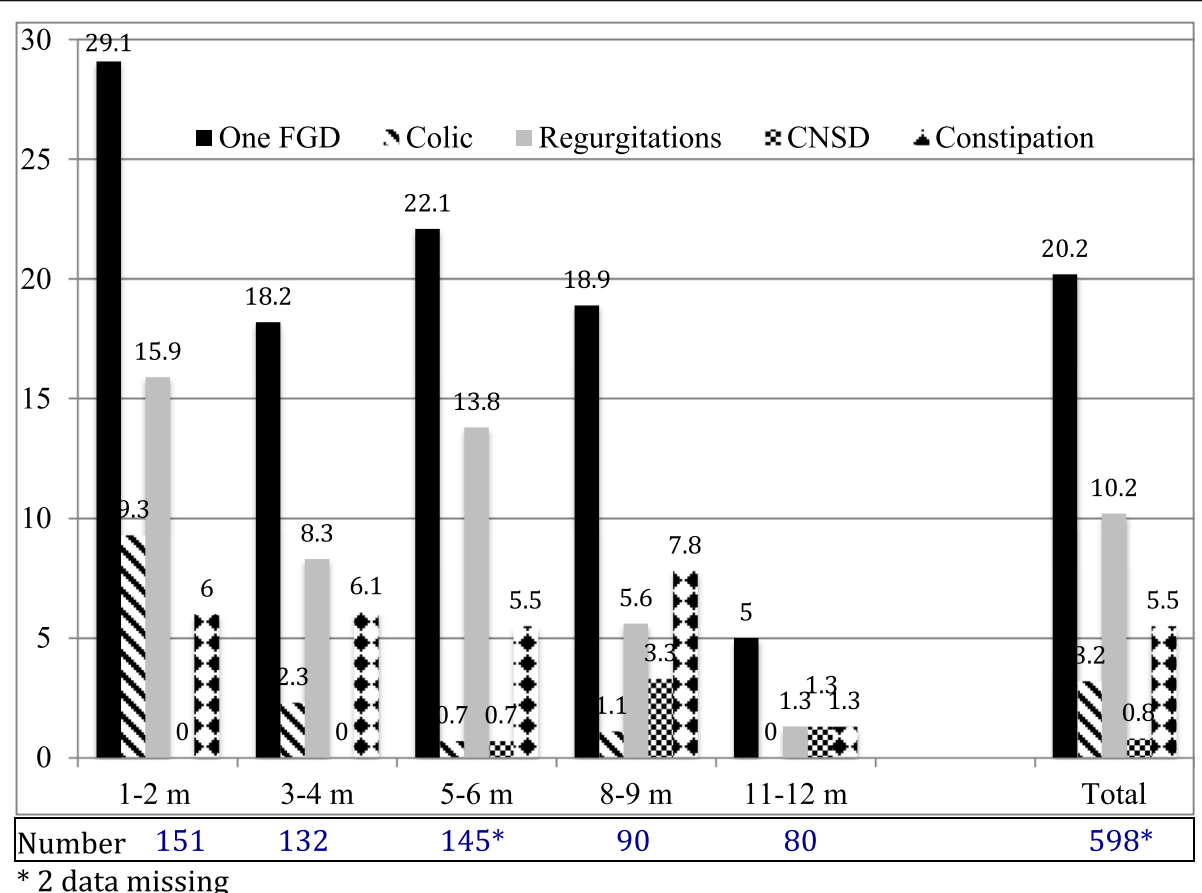

Fig. 1 Prevalence (percentage) of functional gastrointestinal disorders (FGID) in the infants $(n=598)$ at inclusion, which included at least one type of FGID, colic, regurgitation, chronic non-specific diarrhoea (CNSD), and constipation 


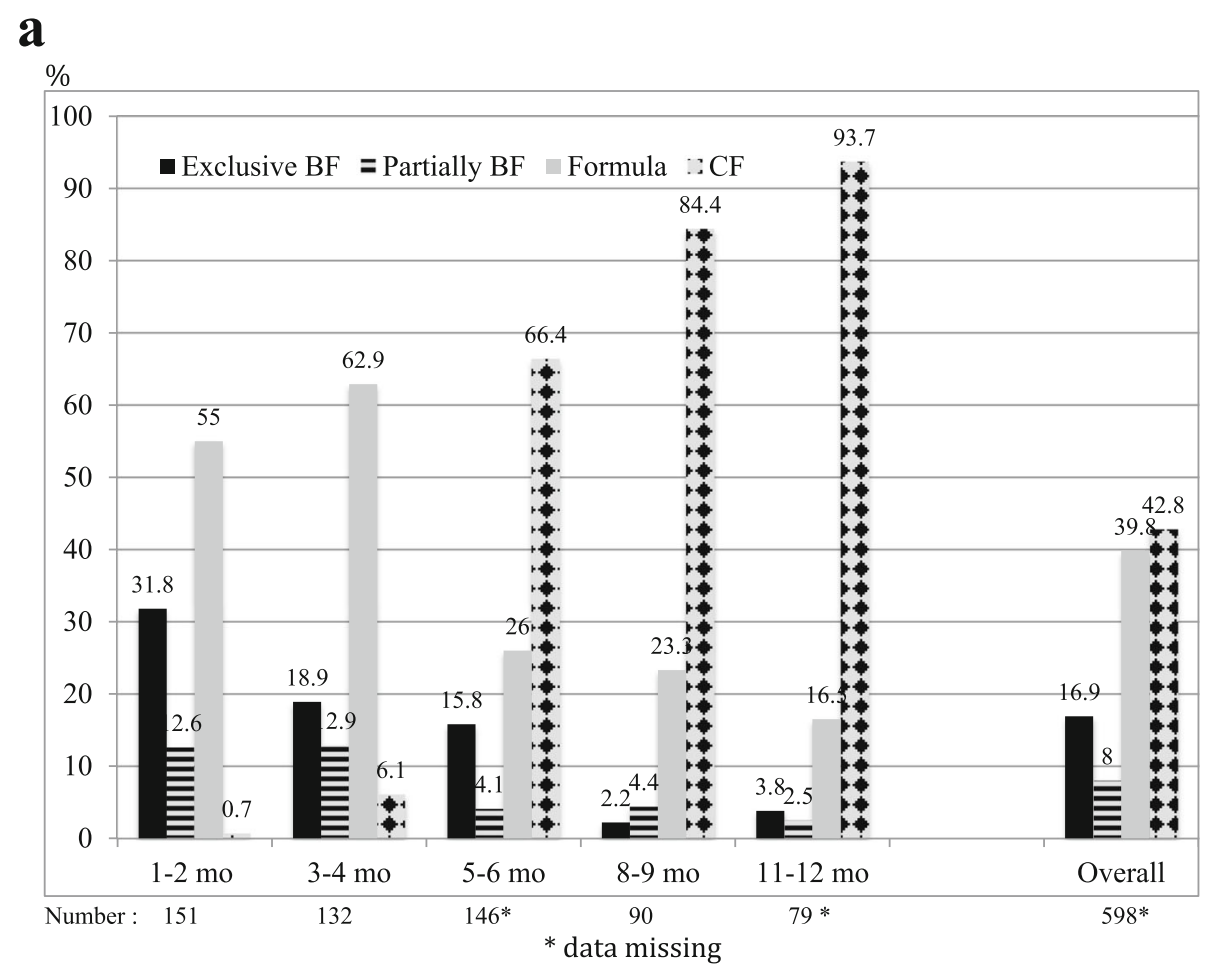

b

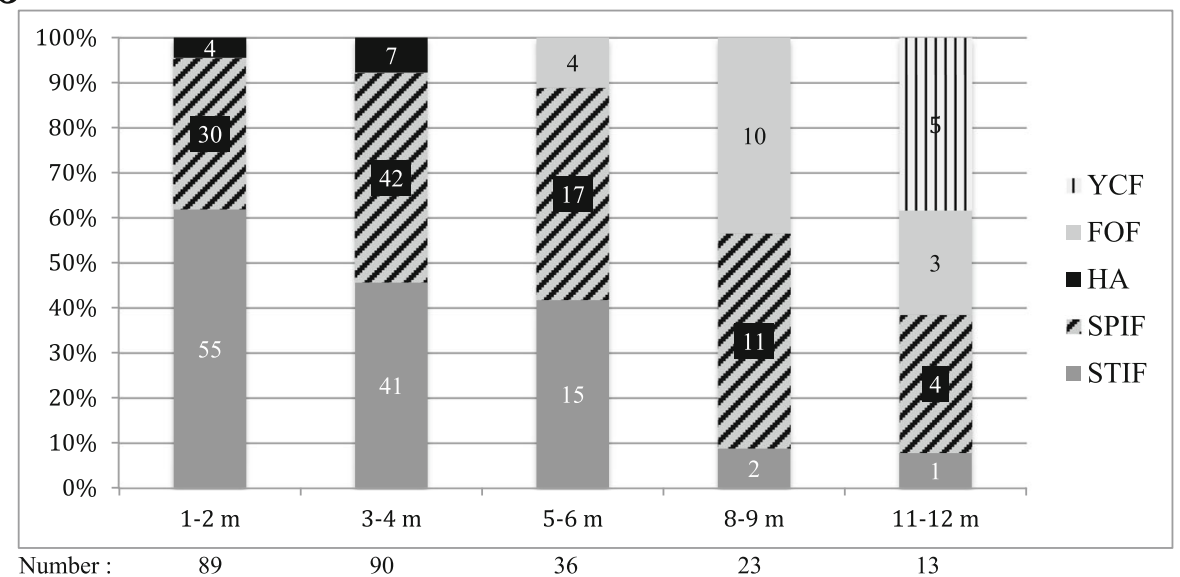

Fig. 2 Feeding practices at inclusion: (a) percentage of infants in the different age groups who were either exclusively breastfed (BF), partially breastfed, or formula fed, and those who consumed complementary foods (CF) including cow's milk and excluding formula and water ( $n=598)$ $[2,6]$, and (b) the prevalence of the consumption of each category of formula in formula-fed infants, when indicated $(n=251)$. The types are identified as standard infant formula (STIF), specific infant formula (SPIF), hypoallergenic formula (HA), follow-on formula (FOF), and young child formula (YCF). The data labels represent the number of infants

(83.2\%) or together with breastfeeding. After 2 months of age, an increasing proportion of infants were fed cow's milk (Table 2). The type of formula used by $87.8 \%$ of the infants is detailed in Fig. 2b; there was no difference between physicians. More than 40\% (115) were fed a specific infant formula designed for either FGID (regurgitations: 36; colic: 3; constipation: 15; satiety: 8; miscellaneous: 31 ), low birth weight (4), prevention of an allergy (HA formula: 11), or suspected cow's milk allergy (7).

\section{Medical advice about feeding}

Medical advice given to $97.8 \%$ of the parents and the time spent doing so are presented in Fig. 3a; the time spent was longer for paediatricians (Fig. 3b). Time spent was considered insufficient by $8.7 \%$ of the physicians, 
Table 2 Comparison of milk diet practices between inclusion (I) and follow up (F). Results are given as number and percentage

\begin{tabular}{|c|c|c|c|c|c|c|c|c|c|c|c|c|}
\hline \multirow{2}{*}{$\begin{array}{l}\text { Age } \\
\text { group }\end{array}$} & \multicolumn{2}{|l|}{$1-2 \mathrm{mo}$} & \multicolumn{2}{|l|}{ 3-4 mo } & \multicolumn{2}{|l|}{$5-6 \mathrm{mo}$} & \multicolumn{2}{|l|}{ 8-9 mo } & \multicolumn{2}{|l|}{$11-12 \mathrm{mo}$} & \multicolumn{2}{|l|}{ Overall } \\
\hline & I & $\mathrm{F}$ & I & $F$ & I & $\mathrm{F}$ & I & $\mathrm{F}$ & I & $\mathrm{F}$ & I & $\mathrm{F}$ \\
\hline Number & 151 & 105 & 132 & 102 & 146 & 111 & 90 & 63 & 79 & 56 & 598 & 437 \\
\hline $\begin{array}{l}\text { Exclusive } \\
\mathrm{BF}\end{array}$ & $48(31.8 \%)$ & $28(26.7 \%)$ & 25 (18.9\%) & 17 (16.7\%) & $23(15.8 \%)$ & 19 (17.1\%) & $2(2.2 \%)$ & $5(7.9 \%)$ & $3(3.8 \%)$ & $3(5.4 \%)$ & 101 (16.9\%) & 72 (16.5\%) \\
\hline Partial BF & 19 (12.6\%) & 15 (14.3\%) & 17 (12.9\%) & 15 (14.7\%) & $6(4.1 \%)$ & $9(8.1 \%)$ & $4(4.4 \%)$ & $3(4.8 \%)$ & $2(2.5 \%)$ & $4(7.1 \%)$ & 48 (8.0\%) & $46(10.5 \%)$ \\
\hline Formula & 83 (55.0\%) & 61 (58.1\%) & $82(62.1 \%)$ & $69(67.6 \%)$ & 38 (26.0\%) & $80(72.1 \%)$ & 21 (23.3\%) & 53 (84.1\%) & 13 (16.5\%) & 46 (82.1\%) & 238 (39.8\%) & $309(70.7 \%)$ \\
\hline CM & $1(0.7 \%)$ & $1(1.0 \%)$ & $8(6.1 \%)$ & $1(1.0 \%)$ & 79 (54.1\%) & $2(1.8 \%)$ & $63(70 \%)$ & $0(0.0 \%)$ & 61 (77.2\%) & $2(3.6 \%)$ & $211(38.5 \%)$ & $6(1.4 \%)$ \\
\hline$p$ & NS & & NS & & $<0.0001$ & & $<0.0001$ & & $<0.0001$ & & $<0.0001$ & \\
\hline
\end{tabular}

BF breast_feeding, CM Cow's milk, mo months

with paediatricians wishing for $15 \min (10-20, n=38)$ and GPs for $10 \min (5-15, n=13)(p=0.001)$.

Breastfeeding advice (Table 3) did not differ between physicians but paediatricians more frequently gave advice about using infant formula (107 vs $16, p=0.01)$. Of the 121 recommendations given for choosing a formula, $43.8 \%$ concerned a specific infant formula (i.e., thickened formula $47.2 \%$, formula for constipation $20.8 \%$, and formula for colic 15.1\%). Advice about introduction of solid foods was given to $59 \%$ of parents (Table 3), and given more frequently by paediatricians $(62.3 \%$ vs $48.2 \%, p=0.002)$.

The recommended age for introduction of solid foods was between 4 and 6 months in $85.2 \%$ of cases. Cooked vegetables were the first category introduced (76\%), followed by cooked fruits $(5.6 \%)$, or both $(14.8 \%)$, and infants' cereals were recommended in only $1.5 \%$ of cases.
Spoon-feeding was the recommended mode of introduction in most cases (89.2\%). Advice about quantities and gradual increases was more common for vegetables or fruit (84.4\%) than for meat or fish (57.1\%). Of 157 pieces of advice about the type of food to give, $40.8 \%$ favoured homemade meals, $13.4 \%$ specific commercial baby foods, and $45.9 \%$ suggested both. For meal preparation, $86.3 \%$ of the advice recommended limiting added salt, 69.3\% was about limiting added sucrose, $49.5 \%$ was about cooking method, and $25.9 \%$ was related to washing vegetables and fruits. In the case of food refusal, parents were advised 'do not force' (60.6\% of 208 recommendations) or 'offer the disliked food repeatedly several times in the following days' (54.3\%).

When gluten introduction was addressed, the main advice $(93.6 \%)$ concerned optimal age, which was between

a

b

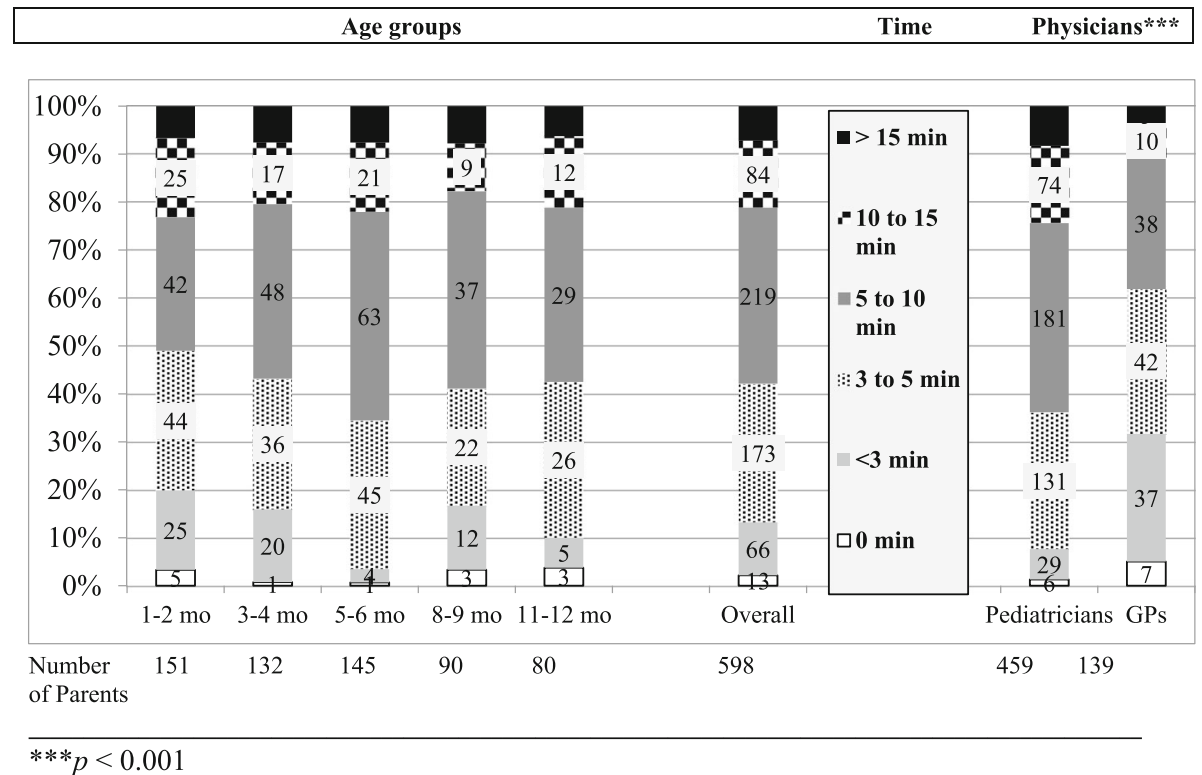

Fig. 3 Time (minutes; min) dedicated to feeding advice: (a) by age group (months; mo) and (b) by physician type (paediatricians or general practitioners (GPs)). The results are given as the percentage of parents to whom time was devoted in each time range. The numbers in the labels represent the number of parents 
Table 3 Physicians advice topics as given to breastfed (BF) or formula fed infants' parents, and about solid foods. Topics given as number and (percentage) of advices

\begin{tabular}{|c|c|}
\hline \multicolumn{2}{|l|}{ Breastfed infants $(n=149)$} \\
\hline Advices given & $100(67.1 \%)$ \\
\hline \multicolumn{2}{|l|}{ Topics (\% of advices) } \\
\hline Feeding frequency & $57(57.0 \%)$ \\
\hline Duration of each feeding & $36(36.0 \%)$ \\
\hline Benefits of BF & $32(32.0 \%)$ \\
\hline Intended weaning & $45(45.0 \%)$ \\
\hline Maternal diet & $26(26.0 \%)$ \\
\hline $\begin{array}{l}\text { Consequences of smoking, } \\
\text { taking alcohol, drugs or medications. }\end{array}$ & 19 (19.0\%) \\
\hline \multicolumn{2}{|l|}{ Formula fed infants $(n=286)$} \\
\hline Advices given & $123(43.0 \%)$ \\
\hline \multicolumn{2}{|l|}{ Topics (\% of advices) } \\
\hline Kind of formula & $122(98.4 \%)$ \\
\hline Volume/bottle & $108(87.8 \%)$ \\
\hline Feeding frequency & $89(72.4 \%)$ \\
\hline Volume/day & $78(63.4 \%)$ \\
\hline \multicolumn{2}{|l|}{ Solid foods $(n=598)$} \\
\hline Advices given & $353(59 \%)$ \\
\hline \multicolumn{2}{|l|}{ Topics (\% of advices) } \\
\hline Age of introduction of solid foods & $223(63.2 \%)$ \\
\hline Order of introduction & $196(55.5 \%)$ \\
\hline Modes of administration & $194(54.5 \%)$ \\
\hline Progressive texture & $256(72.5 \%)$ \\
\hline Quantity of each type of food & $294(83.3 \%)$ \\
\hline Home-made or specific baby-food & $157(44.5 \%)$ \\
\hline Varied diet & $266(75.4 \%)$ \\
\hline Sharing meal with family & $94(26.6 \%)$ \\
\hline Behavior in case of food refusal & $208(58.9 \%)$ \\
\hline Age of introduction of Gluten & $166(47 \%)$ \\
\hline
\end{tabular}

4 and 6 months in $31 \%$ of cases, 4 and 7 months in $23.9 \%$, and after 6 months in $44.5 \%$. The mode of introducing gluten was addressed in $88 \%$ of cases, by adding cereals to the bottle in $58.9 \%$, and by gradually increasing quantity in $80.7 \%$. Postponing introduction of solid foods was recommended for $27.7 \%$ of infants with a parental allergy history, mainly for tropical fruits (61.3\%) or nuts $(23.1 \%)$.

There were differences in the number of paediatricians and GPs providing advice relating to introduction of solid foods mode $(p<0.001)$, food texture ( $77.6 \%$ vs $50.7 \%, p<0.001)$, food quantity $(73.4 \%$ vs $46.3 \%, p=0.046)$, response to food refusal $(64.3 \%$ vs $36.4 \%, p<0.001)$, and introducing gluten $(50.3 \%$ vs $32.8 \%, p=0.01)$.
Among the 54 physicians, 49 (90.7\%) wanted more information about infant feeding topics listed in Table 4; $64 \%$ preferred access via a journal, $44 \%$ via a newsletter, $38 \%$ via the Internet, $20 \%$ via a company document, $8 \%$ via a smartphone app, and some chose several options.

\section{Parents' perceptions and adherence to medical advice} Four hundred and thirty-seven parents $(72.8 \%)$ completed their questionnaire an average of 16 days (14-26) after the initial visit.

\section{Parents' perceptions}

Among these, 434 (99.3\%) answered the questions about medical advice, with 414 (95.4\%) reporting they received one or more pieces of dietary advice and $43 \%$ obtaining a brochure that mainly explained the introduction of solid foods. Overall, the parents' statements about receiving feeding advice were discordant with the physicians' statements $(p<0.0001, \mathrm{~K}=0.11) ; 14.3 \%$ claimed to have received advice that was not reported as given by the physician. This discrepancy was significant in the groups with infants under 5 months. The comparison of the time spent in consultation reported by the parents and that declared by the physician was concordant in $28.9 \%$ of the parents, whereas $33.6 \%$ thought that this time was longer and $37.4 \%$ thought that it was shorter. Of the parents, $84.4 \%$ reported that the consultation time was sufficient versus $91.3 \%$ of the physicians, and there was no concordance between the answers $(p<0.0001, \mathrm{\kappa}=0.079)$.

\section{Parents' adherence}

When all advice was combined, $4.6 \%$ of the counselled parents said they had not followed the advice given so far, $8.7 \%$ had followed it partially, $45.3 \%$ had followed it mostly, and $41.3 \%$ had followed it totally. The parents of younger infants were the most adherent $(p<0.001)$. In descending order, the five most followed pieces of advice were the age for introduction of solid foods, the order in which to introduce the solid foods categories, the choice of formula, food quantity at each age, and food category amounts. About 75\% parents reported that advice they received resulted in improved condition in FGID infants. The most frequent reason parents gave for non-adherence was that it was not suitable for their infant ( $54.4 \%$ of the 22 pieces of infant formula advice not followed and $42.9 \%$ of the 91 pieces of introduction of solid foods advice not followed). The second reason was personal choice followed by conflicting advice from family and friends. The five least followed pieces of advice were about weaning, the amount of formula, the advantage of homemade food, the advantage of specific baby food, and the age for introducing gluten. 
Table 4 Topics of interest about which physicians would like to obtain more information $(n=49)$ shown as the number and percentage of requests

\begin{tabular}{|c|c|c|}
\hline Topics & $\begin{array}{l}\text { Number of requests (\%) } \\
\text { for the information }\end{array}$ & $\begin{array}{l}\text { Number of requests } \\
\text { (\%) first cited }\end{array}$ \\
\hline Scientific societies' guidelines & $27(50.0)$ & $12(24.5)$ \\
\hline Diet of infants with FGID & $27(50.0)$ & $2(4.1)$ \\
\hline Nutrient requirement & $26(46.3)$ & $12(24.5)$ \\
\hline Prevention of allergy & $21(38.9)$ & $8(16.3)$ \\
\hline Protein requirement & $18(33.3)$ & $4(8.2)$ \\
\hline Food introduction/age & $18(33.3)$ & $2(4.1)$ \\
\hline Textures/age & $16(29.6)$ & $1(2.0)$ \\
\hline Gluten introduction & $14(25.9)$ & $1(2.0)$ \\
\hline Treatment of food allergy & $14(25.9)$ & $2(4.1)$ \\
\hline Quantities of milk/age & $9(16.7)$ & $4(8.2)$ \\
\hline Weaning & $7(13.0)$ & $1(2.0)$ \\
\hline
\end{tabular}

FGID functional gastrointestinal disorders

\section{Dietary practices at the follow-up}

Following the consultation, the consumption of cow's milk by infants older than 5 months was significantly reduced, replaced by more frequent use of formula (Table 2).

\section{Discussion}

Since few studies [9-12, 15, 16, 19-23] have conducted similar investigations, this pilot study provides important data on differences between professionals' perceptions and parents' perceptions and subsequent behaviour in regard to infant feeding, and highlights a number of key issues important to developing future guidelines. Except for one earlier study [19] the current study is the only one to recruit both paediatricians and GPs.

Although it is not certain that the Rome III criteria were applied strictly, the prevalence of FGID in our sample was much lower than previously reported [24, 25]. The GPs higher proportion of FGID infants may be due to patient and family differences but may also be a result of poor knowledge of the criteria [18]. The prevalence of parental atopic history is higher than that originally described in one study (14.3\%) [17], but similar or even lower than in more recent studies (27.2 and 43.2\%) [26, 27].

This survey recognised that the majority of physicians and parents were aware of infant feeding methods. However, initial dietary practices in our sample were far from optimal regarding breastfeeding and the preference for infant formula instead of cow's milk [2-6]. No information was collected about what may have influenced these practices. The prevalence of breastfeeding is generally reported as low in France, [16, 28, 29]. A surprisingly large proportion of non-breastfed infants in our sample had already consumed cow's milk, which was higher than that reported in the Nutri-Bébé study (5.1\%) [16], and less than half of the infants consumed a formula. Of note is the small number of children who were fed HA formula considering the observed prevalence of family allergy history, but at-risk infants were not identified among the breastfed infants. This contrasts with the results of the AllerNaiss study reporting a screening strategy for allergy history in 59\% of 387 French maternity wards and a subsequent prescription of HA formula, in the absence of breastfeeding in $90 \%$ of the wards [30].

The majority of parents adhered to the guidelines when introducing solid foods. No infant younger than 5 months consumed them. In the Nutri-Bébé study, the mean age for the introduction of solid foods was $5.4 \pm$ 2.1 months [16]. These results are more encouraging than those reported by other researchers [9, 11, 19]. Of note, however, is the persistence of postponing introducing some specific foods in case of parental allergy history. Information given about texture was more frequent than previously reported [22].

Most of the physicians spent time giving advice. Overall, this advice was in line with the guidelines, whereas in a study conducted in southern France, only $65 \%$ of paediatricians $(n=50)$ or paediatric residents $(n=34)$ gave such advice [21]. Physicians would like to obtain more information about infant feeding practices, which highlights the gap between the publication of scientific guidelines and professionals' perception.

In the follow-up, the parents' response rate was high and very few said they were not observing the advice. As a result, the main deviance initially observed, the early consumption of cow's milk, was corrected. Among noncompliant parents, the reason given was lack of confidence in their doctor and a preference for advice from family and friends or other environmental sources $[15,19,20]$. It is astonishing to note that the advantages of homemade 
food or specific baby food and the age for introducing gluten were among the least followed pieces of advice. There is a large discrepancy globally between physicians' statements and parents' perceptions. While reported compliance with current infant feeding guidelines was not terribly poor, there is nevertheless a long way to go before the behaviour perfectly matches the guidelines. As proposed by others, the parents' and physicians' interest in nutrition and health, coupled with their confusion, suggests that there are shortcomings in current nutrition communication and that there are important opportunities to improve dietary guidance $[13,31]$.

Our results should be viewed in the context of the study's limitations. The number of participating physicians was relatively small, but they recruited a larger sample of parents than in other studies [9, 15, 20, 23]. We acknowledge that our survey recruited a selected and demographically biased sample of parents who were mostly educated and fluent in French and able to use the Internet, which is not nationally representative. Moreover, only primiparous mothers were recruited, and having more children is linearly associated with higher nutritional knowledge $(p<0.01)$ [10]. Our results are also based on self-reported practices rather than objective audit data, which may compromise reliability. Finally, while using a structured questionnaire allowed precise and comparable answers, it may also have guided the answers.

\section{Conclusion}

While our study does not reveal a large gap between current guidelines and physicians' and parents' practices, a number of improvements are desirable. Given the study's declarative aspects conclusions must be interpreted with caution. However, an important point was raised-despite the existence of several published guidelines, some professionals and parents seek nutrition information while others ignore the recommendations, emphasising the need to review strategies for promoting good nutrition. Providing consistent and relevant advice might decrease confusion and render the advice more acceptable and increasing awareness of media influences may decrease messages that conflict with scientific committees' guidelines.

Indeed possible contradictions between opinion leaders lead to confusion, which contributes to counteracting nutrition counselling in general and lead parents to consider scientists really do not know what foods are good for their child. But what matters is that dietary advice must clearly explain the recommended behaviours and why changes have occurred so that consumers have the opportunity to develop an appreciation of how scientific knowledge evolves. Effective guidance needs to consider what really matters to parents and must promote recommendations based on foods instead of nutrients. One step could be to help parents understand why and how healthy eating can be beneficial in the short, medium and long term for their child. Nutrition messages need to be simple, clear, easy to understand, realistic, positive, and exploitable fitting with modern lifestyles without demanding a complete renunciation of habits. An objective audit of medical practices is needed but may be difficult to achieve.

\section{Additional files}

Additional file 1: Physician questionnaires including birth and current infant characteristics; parents' allergy history; functional gastrointestinal disorders; infant feeding practices since birth and currently; dietary advice; time spent providing the advice. (PDF $181 \mathrm{~kb}$ )

Additional file 2: Parent questionnaires including infant feeding at follow-up, adherence to the doctor's advice, observance in their daily practice, and their feelings about the time devoted by the physician to the advice. (DOC $92 \mathrm{~kb}$ )

\section{Abbreviations}

BF: Breast-feeding; CNSD: Chronic non-specific diarrhoea; d: day; FGID: Functional gastrointestinal disorders; FOF: Follow-on formula; GP: General practitioners; HA: Hypoallergenic formula; IF: Infant formula; ISF: Introduction of solid foods; SPIF: Specific infant formula; STIF: Standard infant formula; YCF: Young child formula

\section{Acknowledgements}

The authors thank all the physicians and families who gave their time to participate in the survey as well as the data management and statistical analysis team.

\section{Authors' contributions}

JPC analysed the data, wrote the manuscript and was responsible for the final content; $\mathrm{BD}$ collaborated in analysing data; $\mathrm{ML}, \mathrm{MB}, \mathrm{CJ}$ and $\mathrm{TH}$ collaborated in the design and the execution of the study. All authors revised the manuscript's final content. All authors read and approved the final manuscript.

\section{Funding}

The survey was fully funded by Nestlé SA. Three employees of this company participated in the design and the execution of the study and revised the final manuscript without intervening in the interpretation of the results nor the conclusions. Their individual contribution are indicated in Author's contributions.

\section{Availability of data and materials}

All data generated and analysed during this study are included in this published article. Nevertheless, the datasets used and/or analysed during the current study are available, on reasonable request, from the following research organization: Stat Process, 52, Boulevard Sébastopol, 75003 Paris, France. florence.mercier@statprocess.com

\section{Ethics approval and consent to participate}

The entire survey was in accordance with the 1964 Helsinki declaration and it's later amendments. According to Article R1121-1-II-2 of the French Public Health Code, the survey designed to evaluate the exercise of health professionals and which does not involve any medication or blood tests are exempt from ethical review (https://www.legifrance.gouv.fr/telecharger_pdf. do?cidTexte=LEGITEXT000006072665). The study protocol and the online procedures were approved by the Comité Consultatif sur le Traitement de I'Information en Matière de Recherche dans le Domaine de la Santé (Advisory Committee on Information Processing for Health Research; reference number: CCTIRS 15343, 13 May2015).

Informed consent was obtained from all individual parents included in the study first verbally by the physician, then in writting on line when parents first connected. 


\section{Consent for publication}

Not applicable.

\section{Competing interests}

JPC received fees from Nestlé as a consultant and for giving lectures and received support from Nestlé and Mead Johnson to attend a congress. MB and $\mathrm{CJ}$ received fees from Nestlé in the context of this study and from Laudavie unrelated to this study. MB also received fees from Danone and Lactalis as a consultant and for giving lectures. BD, ML, and $\mathrm{TH}$ are all employed by Nestlé

\section{Author details}

${ }^{1}$ Paediatric Nutrition and Gastroenterology, Division of Pediatrics, Woman, Mother and Child Department, Centre Hospitalier Universitaire Vaudois (CHUV), 21 rue du Bugnon, 1011 Lausanne, Switzerland. Nestlé, Département Médical et Nutrition Infantile, 7 Boulevard Pierre Carle, BP 900 Noisiel, France. ${ }^{3}$ Service de Gastroentérologie, Mucoviscidose et Nutrition Pédiatriques, Hôpital Robert Debré, 48 Boulevard Serurier, 75935 Paris, France. ${ }^{4}$ Centre de Recherche Clinique, $\mathrm{CHI}$ Creteil, 40 Avenue de Verdun, 94000 Creteil, France.

Received: 25 March 2019 Accepted: 27 August 2019 Published online: 04 September 2019

\section{References}

1. Simeoni U, Bocquet A, Briend A, Chouraqui J-P, Darmaun D, Dupont C, et al. Comité de nutrition de la Société française de pédiatrie. [Early origins of adult disease]. Arch Pediatr. 2016;23:443-6.

2. EFSA Panel on Dietetic Products, Nutrition and Allergies (NDA). Scientific Opinion on the appropriate age for introduction of complementary feedingof infants. EFSA J. 2009;7(12):38. https://doi.org/10.2903/j.efsa.2 009.1423 Available from: www.efsa.europa.eu/efsajournal.

3. EFSA NDA Panel (EFSA Panel on Dietetic Products, Nutrition and Allergies). Scientific Opinion on nutrient requirements and dietary intakes of infants and young children in the European Union. EFSA J. 2013;11(10):103. https://doi.org/10.2903/j.efsa.2013.3408 Available from: www.efsa.europa.eu/efsajournal.

4. EFSA NDA Panel (EFSA Panel on Dietetic Products, Nutrition and Allergies). Scientific Opinion on the essential composition of infant and follow-on formulae. EFSA J. 2014;12(7):106. https://doi.org/10.2903/j.efsa.2014.3760 Available from: www.efsa.europa.eu/efsajournal.

5. Turck D, Dupont C, Vidailhet M, Bocquet A, Briend A, Chouraqui JP, et al. Comité de nutrition de la Société Française de Pédiatrie. [Complementary feeding: Evolving concepts and recommendations]. Arch Pediatr. 2015;22:457-60.

6. World Health Organization. Infant and young child feeding: model chapter for textbooks for medical students and allied health professionals. Fact sheets 2018. Available from https://www.who.int/news-room/fact-sheets/ detail/infant-and-young-child-feeding. Accessed 13 Feb 2019.

7. Szajewska H, Shamir R, Mearin L, Ribes-Koninckx C, Catassi C, Domellöf M, et al. Gluten Introduction and the Risk of Coeliac Disease: A Position Paper by the European Society for Pediatric Gastroenterology, Hepatology, and Nutrition. J Pediatr Gastroenterol Nutr. 2016;62:507-13.

8. Fewtrell M, Bronsky J, Campoy C, Domellöf M, Embleton N, Fidler Mis N, et al. Complementary Feeding: A Position Paper by the European Society for Paediatric Gastroenterology, Hepatology, and Nutrition (ESPGHAN) Committee on Nutrition. J Pediatr Gastroenterol Nutr. 2017;64:119-32.

9. Synnott K, Bogue J, Edwards CA, Scott JA, Higgins S, Norin E, et al. Parental perceptions of feeding practices in five European countries: an exploratory study. Eur J Clin Nutr. 2007;6:946-56.

10. Wojcicki JM, Gugig R, Kathiravan S, Holbrook K, Heyman MB. Maternal knowledge of infant feeding guidelines and label reading behaviours in a population of new mothers in San Francisco, California. Matern Child Nutr. 2009:5:223-33.

11. Allcutt C, Sweeney MR. An exploration of knowledge, attitudes and advice given by health professionals to parents in Ireland about the introduction of solid foods. A pilot study. BMC Public Health. 2010;10:201 Epub 2010 Apr 21.

12. O'Donovan SM, Murray DM, Hourihane JO, Kenny LC, Irvine AD, Kiely M. Adherence with early infant feeding and complementary feeding guidelines in the Cork BASELINE Birth Cohort Study. Public Health Nutr. 2015;18:2864-73.
13. Webb D, Byrd-Bredbenner C. Overcoming consumer inertia to dietary guidance. Adv Nutr. 2015;6:391-6.

14. Huysentruyt K, Laire D, Van Avondt T, De Schepper J, Vandenplas Y. Energy and macronutrient intakes and adherence to dietary guidelines of infants and toddlers in Belgium. Eur J Nutr. 2016;55:1595-604.

15. Russell CG, Taki S, Azadi L, Campbell KJ, Laws R, Elliott R, et al. A qualitative study of the infant feeding beliefs and behaviours of mothers with low educational attainment. BMC Pediatr. 2016;16:69.

16. Chouraqui JP, Tavoularis $G$, Emery $Y$, Francou A, Hébel $P$, Bocquet $M$, et al. The French national survey on food consumption of children under 3 years of age - Nutri-Bébé 2013: Design, methodology, population sampling and feeding practices. Public Health Nut. 2018;21:502-14.

17. Kjellman NIM. Atopic disease in seven-year-old children. Incidence in relation to family history. Acta Paediatr Scand. 1977;66:465-71.

18. Hyman PE, Milla PJ, Benninga MA, Davidson GP, Fleisher DF, Taminiau J. Childhood functional gastrointestinal disorders: neonate/toddler. Gastroenterol. 2006;130:1519-26.

19. Moore AP, Milligan P, Goff LM. An online survey of knowledge of the weaning guidelines, advice from health visitors and other factors that influence weaning timing in UK mothers. Matern Child Nutr. 2014;10:410.

20. Duncanson K, Burrows T, Holman B, Collins C. Parents' perceptions of child feeding: a qualitative study based on the theory of planned behavior. J Dev Behav Pediatr. 2013;34:227-36.

21. Banti T, Carsin A, Chabrol B, Reynaud R, Fabre A. Infant food diversification. Assessment of practices in relation to French recommendations in pediatricians and pediatric residents in southern France. Arch Pediatr. 2016;23:1018-27.

22. Marduel Boulanger A, Vernet M. Introduction of new food textures during complementary feeding: Observations in France. Arch Pediatr. 2018;25:6-12.

23. Appleton J, Laws R, Russell CG, Fowler C, Campbell KJ, Denney-Wilson E. Infant formula feeding practices and the role of advice and support: an exploratory qualitative study. BMC Pediatr. 2018;18:12.

24. Vandenplas $Y$, Abkari A, Bellaiche M, Benninga M, Chouraqui JP, Çokura $F$, et al. Prevalence and health outcomes of functional gastrointestinal symptoms in infants from birth to 12 months of age. J Pediatr Gastroenterol Nutr. 2015;61:531-7.

25. Robin SG, Keller C, Zwiener R, Hyman PE, Nurko S, Saps M, et al. Prevalence of Pediatric Functional Gastrointestinal Disorders Utilizing the Rome IV Criteria. J Pediatr. 2018;195:134-9.

26. Pyrhönen $K$, Hiltunen L, Kaila M, Näyhä S, Läärä E. Heredity of food allergies in an unselected child population: an epidemiological survey from Finland. Pediatr Allergy Immunol. 2011;22:e124-32.

27. Pohlabeln H, Mühlenbruch $\mathrm{K}$, Jacobs $\mathrm{S}$, Böhmann $\mathrm{H}$. Frequency of allergic diseases in 2-year-old children in relationship to parental history of allergy and breastfeeding. J Investig Allergol Clin Immunol. 2010;20:195-200.

28. Salanave B, De Launay C, Boudet-Berquier J, Castetbon K. Durée de l'allaitement maternel en France (Épifane 2012-2013). Bull Epidémiol Hebd. 2014;27:450-7 Available from: http://www.invs.sante.fr/beh/2014/27/2014_27_2.html.

29. Wagner S, Kersuzan C, Gojard S, Tichit C, Nicklaus S, Geay B, et al. Durée de l'allaitement en France selon les caractéristiques des parents et de la naissance. Résultats de l'étude longitudinale Française Elfe, 2011. Bull Epidémiol Hebd. 2015;29:522-32 Available from: http://www.invs.sante.fr/ beh/2015/29/2015_29_1.html.

30. Chouraqui JP, Simeoni U, Tohier C, Nguyen F, Kempf C, Beck L, et al. Screening for the risk of allergy and prevention in French maternity units: $A$ survey. Arch Pediatr. 2015;22:943-50.

31. Watts ML, Hager MH, Toner CD, Weber JA. The art of translating nutritional science into dietary guidance: history and evolution of the Dietary Guidelines for Americans. Nutr Rev. 2011;69:404-12.

\section{Publisher's Note}

Springer Nature remains neutral with regard to jurisdictional claims in published maps and institutional affiliations. 